\title{
The analysis of blood proteins by ESI-mass spectrometry for endogenous mental disorders
}

\author{
A.A. Seregin ${ }^{1 *}$, L.P. Smirnova ${ }^{1}$, E.M. Dmitrieva ${ }^{1}$, A.A. Letova ${ }^{2}$, L.V. Loginova ${ }^{1}$, \\ G.G. Simutkin ${ }^{1}$, S.A. Ivanova ${ }^{1}$, V. Zgoda ${ }^{3}$ \\ ${ }^{1}$ Mental Health Research Institute, Tomsk National Research Medical Center of the Russian Academy \\ of Sciences, Tomsk, Russia \\ ${ }^{2}$ Siberian State Medical University, Tomsk, Russia \\ ${ }^{3}$ Institute of Biomedical Chemistry (IBMC) \\ *e-mail:apocalips1991@mail.ru
}

Key words: Bipolar disorder, protein markers, proteomics, blood serum

Motivation and Aim: Bipolar affective disorder (BD) refers to endogenous disorders and is similar to schizophrenia; it develops without the influence of external factors. However, their biological differences are not revealed. Nowadays proteomic studies of the blood composition in patients with BD via ESI-mass spectrometry are missing.

Methods and Algorithms: In this work, serum of 8 patients with BD and 5 healthy individuals were analyzed. The samples were subjected to affinity chromatography, 1D SDS page and in-gel trypsinolysis of the proteins. The mass-spectrometric analysis was carried out via mass spectrometer-Thermo Scientific LTQ Velos. Identification of proteins was carried out via Matrix Science resources. Statistical significance was assessed by Fisher exact test with the Yates correction, $p<0.05$.

Results: As a result of the analysis, from 150 to 500 proteins were identified in every sample. Proteins, presenting in BD, are mostly involved in the processes of cell growth and maintenance, regulation of nucleic acid metabolism, followed by the immune response, protein metabolism and unknown processes. Including detected: Plectin with a detection rate of healthy $20 \%$, in patients - $50 \%$; Vimentin - detection rate in patients $-75 \%$, of healthy is not found; Glutamate ionotropic receptor NMDA type subunit 1 (NMDAR) - frequency of detection in patients - $25 \%$, not detected in the control; Unconventional myosin-Va - detection rate in healthy $20 \%$, in patients $-75 \%$; $\mathrm{SH} 3$ and multiple ankyrin repeat domains protein 1 - frequency of detection in patients $37.5 \%$, not detected in the control group.

Conclusion: SH3 and multiple ankyrin repeat domains protein 1, as a cytoskeleton protein, represents a great interest because it connects postsynaptic membrane receptors including metabotropic glutamate and NMDA receptors with the actin cytoskeleton. That several point mutations are revealed in SHANK gene encoding SH3 and multiple ankyrin repeat domains protein 1 presumably contributing to the development of autism spectrum disorders. Unconventional myosin-Va and other cytoskeletal proteins influence the regulation of NMDAR and are in the blood due to the violation of the permeability of blood-brain barrier.

Acknowledgements: Mass spectrometric analysis was carried out using the equipment of "Human Proteome" Core Facility of the Institute of Biomedical Chemistry (IBMC) Moscow which is supported by Ministry of Education and Science of the Russian Federation (unique project ID RFMEFI62117X0017) Support by Grant of RSF No. 18-15-00053. 